PANORAMA DE LAS CARACTERÍSTICAS DEL CONSUMIDOR DE CERVEZA ARTESANAL EN LA CIUDAD DE MONTERREY, NUEVO LEÓN.

\title{
Panorama de las características del consumidor de cerveza artesanal en la ciudad de Monterrey, Nuevo León
}

\section{Outlook on the characteristics of the craft beer consumer from Monterrey, Nuevo Leon}

María del Socorro Martínez Silva*, Olga Beatriz Sánchez Rosado**

\begin{abstract}
*Estudiante de la Licenciatura en Mercadotecnia. División Académica de Ciencias Económico Administrativas. Universidad Juárez Autónoma de Tabasco. Villahermosa, Tabasco, México. ORCID: https://orcid.org/0000-0002-0163-0827.

**Maestra en Mercadotecnia y Doctora en Psicoterapia Gestalt Relacional. Profesora Investigadora. División Académica de Ciencias Económico Administrativas. Universidad Juárez Autónoma de Tabasco. Email: betytab@hotmail.com. ORCID: https://orcid.org/0000-0003-4845-9382.
\end{abstract}

Correo para recibir correspondencia: maricokey@gmail.com

Fecha de recibido: 19 de agosto de 2020

Fecha de aceptación: 10 de octubre de 2020 
PANORAMA DE LAS CARACTERÍSTICAS DEL CONSUMIDOR DE CERVEZA ARTESANAL EN LA CIUDAD DE MONTERREY, NUEVO LEÓN.

\section{RESUMEN}

La cerveza artesanal en México es un producto relativamente nuevo en un mercado muy competido, por ello la necesidad de conocer el segmento al cual los nuevos emprendedores puedan dirigir sus esfuerzos de marketing. Nuevo León, es uno de los principales estados del país que presenta mayor consumo en bebidas alcohólicas, así como un gran número de cervecerías artesanales, su capital Monterrey, posee características favorables para el desarrollo del presente estudio con enfoque cuantitativo que describe las características que presentan los consumidores actuales de cerveza artesanal.

OBJETIVO: Describir las características que presenta el consumidor de cerveza artesanal en la ciudad de Monterrey, Nuevo León.

MATERIAL Y MÉTODO: La presente investigación cuenta con un enfoque cuantitativo de diseño no experimental, transversal, de alcance descriptivo y de muestra no probabilística.

RESULTADOS: El segmento que mayormente adquiere este producto se encuentra en un rango de edad de 18 a 35 años, en su etapa de soltería, laboran actualmente, se encuentran en un nivel superior de estudios y los lugares preferentes para consumir esta bebida son bares y restaurantes.

CONCLUSIONES: En el caso de la presente investigación, los datos proporcionados, podrán ayudar a crear estrategias de marketing focalizadas para el consumidor de la ciudad de Monterrey, permitiendo a la cervecería obtener reconocimiento principalmente a nivel local.

PALABRAS CLAVE: Consumidor. Cerveza. Cerveza artesanal. Monterrey, Nuevo León.

\section{ABSTRACT}

Craft beer in Mexico is a relatively new product in a very competitive market, hence the need to know the segment so marketing efforts can be directed by new entrepreneurs. Nuevo Leon is one of the main states in the country with a high consumption level of alcoholic beverages, and a great number of craft brewing companies. Monterrey, its 
PANORAMA DE LAS CARACTERÍSTICAS DEL CONSUMIDOR DE CERVEZA ARTESANAL EN LA CIUDAD DE MONTERREY, NUEVO LEÓN.

capital city, has favorable characteristics for the development of this quantitative study in which the characteristics of recent craft beer consumers are described.

OBJECTIVE: To describe the characteristics a craft beer consumer from Monterrey, Nuevo Leon has.

MATERIAL AND METHOD: This is a non-experimental quantitative research, crosssectional design, descriptive scope, and a non-probabilistic sample.

RESULTS: The segment that mostly buys this product is between 18 to 35 years old, and of single status. Currently working, with a diploma, and are likely to visit bars and restaurants where this type of beer is sold.

CONCLUSIONS: In this research, the obtained data may help create strategic marketing efforts for the craft beer consumer profile in Monterrey, thus allowing beer companies to get recognition mainly at a local level.

KEY WORDS: Consumer. Beer. Craft beer. Monterrey, Nuevo León.

\section{INTRODUCCIÓN}

Murray y O'Neill (2012, p. 3) en su estudio sobre cerveza artesanal determinaron que el mercado de cerveza artesanal es creciente, pues las características demográficas como la edad, ingresos y el nivel de estudios que presentaba el consumidor eran suficientes para afirmar que el mercado continuaría en ascenso.

Hoy en día, se puede decir que la cerveza artesanal es un sector que continúa ganando mercado y que en México no es la excepción. Este mercado dentro del país ha cobrado gran fuerza en los años recientes y lo podemos afirmar al conocer los datos proporcionados por la Asociación de Cerveceros Mexicanos (ACERMEX), en el 2018 se registró la apertura de 310 cervecerías artesanales, en total actualmente existen 940, siendo los estados de Jalisco, Baja 
PANORAMA DE LAS CARACTERÍSTICAS DEL CONSUMIDOR DE CERVEZA ARTESANAL EN LA CIUDAD DE MONTERREY, NUEVO LEÓN.

California, Michoacán, Ciudad de México y Nuevo León sede de mayor numero de cervecerías artesanales (ACERMEX, 2019, p. 8).

El surgimiento y esparcimiento de estas cervecerías dentro de la República Mexicana reflejan el mismo aumento de la demanda por parte del consumidor, según Kantar World Panel (2016) "México es el segundo país en Latinoamérica consumidor de cerveza, 7 de cada 10 hogares compraron esta bebida en el 2015" y para el 2017 según Quiroga (2018) "a nivel global el consumo per cápita de los mexicanos fue de 65 litros, colocándonos en el lugar número 32 a nivel mundial".

Cabe destacar, que la importancia de este producto en el país no es solo de consumo, puesto que la producción de cerveza en México según el INEGI (2017, p. 12) se ubica entre las 14 actividades más importantes de la producción manufacturera, de un total de 291. En 2017 según Cerveceros Mexicanos, citado por Calvillo (2017, p. 11) esta industria generó más de 55 mil empleos directos, de los cuales en 2018 las cervecerías artesanales son responsables de 6,373 (ACERMEX, 2019, p. 10).

Urrutia, Aguilar y Muños (2016, p. 380) expresan que en México al igual que en otros países la industria cervecera está determinada por dos compañías que concentran la totalidad del volumen del mercado, la presente investigación nace bajo las siguientes cuestiones: ¿Cómo puede crecer una cervecería artesanal en un mercado tan competitivo? ¿Cuál es el papel del marketing dentro de una cervecería artesanal? ¿Cuáles son las características que presenta el consumidor de cerveza artesanal?

Como se hace mención anteriormente, Nuevo León es uno de los estados sede con mayor número de cervecerías artesanales, de igual manera cabe destacar su lugar dentro de los 10 estados del país con mayor consumo de alcohol (Secretaría de Salud, Instituto Nacional de Salud Pública e Instituto Nacional de Estadística y Geografía, 2018, p. 26). En un estudio realizado por Villezca (2005, p. 343), se explica en específico, en el área metropolitana de Monterrey, al incrementarse el ingreso de las personas, el gasto en consumo de cerveza también se eleva y que la presencia de hombres, entre 20 y 64 años, incrementa significativamente la probabilidad de compra de este bien en todos los estratos de ingreso.

En virtud de lo anterior, la presente investigación tiene como objetivo describir las características que presenta el consumidor de cerveza artesanal, en la ciudad de Monterrey, MARTÍNEZ-SILVA M. DEL S., SÁNCHEZ-ROSADO O. B. 
PANORAMA DE LAS CARACTERÍSTICAS DEL CONSUMIDOR DE CERVEZA ARTESANAL EN LA CIUDAD DE MONTERREY, NUEVO LEÓN.

Nuevo León, con el propósito de que a partir de la misma los nuevos emprendedores puedan conocer el segmento al cual dirigir sus esfuerzos de marketing.

\section{Historia de la cerveza en México}

Al hablar de cerveza en México se pensaría que es una bebida que ha nacido en el país puesto que en la actualidad es el principal exportador de este producto (Workman, 2020), pero años atrás la producción de cerveza comenzó después de la conquista española, se importaba cerveza de Estados unidos, Gran Bretaña y Alemania. Los estados al norte del país eran los principales consumidores de cerveza pues antes de 1890 la mayoría de la población seguía consumiendo bebidas mucho más populares y baratas como el pulque, mezcal y aguamiel (Recio, 2004, p. 5-7).

En los años siguientes a 1890, la industria cervecera se estableció en Monterrey con una de las dos grandes cervecerías mexicanas que hasta la actualidad continúan su expansión a nivel global, la Cervecería Cuauhtémoc-Moctezuma, que en sus comienzos trabajó con maestros cerveceros de Alemania para iniciar su producción en el país (Contreras Delgado \& Ortega Ridaura, 2005, p. 91).

Para 1929, en la ciudad de Monterrey, Nuevo León, el nacimiento de la cervecería Cuauhtémoc había hecho crear otras industrias que permitieron la formación de una expansión vertical, menciona Ortega (2002, p. 9) que en ese año las actividades que no estaban directamente relacionadas con la fabricación de cerveza (ácido carbónico, tapón corona, cajas de cartón), se agruparon bajo la denominación Fábricas Monterrey, aunque siguieron formando parte de la cervecería.

En 1995, a la par del crecimiento de los dos grandes gremios (Cervecería CuauhtémocMoctezuma y Grupo Modelo [fundada en 1925]), en el centro del país nació la primera cervecería artesanal misma que fue inspirada en las cervezas estadounidenses. Cabe recalcar que el concepto de "cervecería artesanal" tiene su origen en el Reino Unido y se denominaba así a la generación de pequeñas cervecerías que se enfocaban en la producción tradicional de cervezas estilo ale (cerveza de fermentación alta) (Calvillo, 2017, p. 8). 
PANORAMA DE LAS CARACTERÍSTICAS DEL CONSUMIDOR DE CERVEZA ARTESANAL EN LA CIUDAD DE MONTERREY, NUEVO LEÓN.

\section{Cerveza artesanal}

En la actualidad, como menciona Urrutia et al. (2016, p. 381) se identifican cuatro categorías de cerveza artesanal, micro cervecería, brewpub (bar-restaurante), cervecería contratista y cervecería artesanal regional, tal como lo establece la Comisión Federal de Competencia (COFECE) ninguna de las clasificaciones anteriores produce más del $1 \%$ del mercado cervecero nacional (¿Qué es una cerveza artesanal?, 2018).

Cabe recalcar, a nivel global la cerveza es una bebida fermentada derivada de la mezcla resultante de malta, lúpulo, agua y levadura (Suárez Díaz, 2013), y que se distingue de una cerveza artesanal por el nivel de producción de la cervecería. De igual manera, un punto focal diferenciador es que los pequeños productores experimentan con distintas especies, frutas y otros ingredientes para crear cervezas que les permita transmitir su personalidad a través de diversos estilos.

\section{Marketing y cervezas artesanales}

Para las cervecerías artesanales," las estrategias de mercadotecnia son diferentes a las de compañías de cerveza industrial, pues ofrecen productos que compiten según su calidad y diversidad, en lugar de precios bajos y publicidad" (Calvillo, 2017, p. 4). En México el mercado de la cerveza artesanal se enfrenta a diversos retos dentro de los cuales el factor precio de venta y la publicidad en medios masivos, representan ser los más grandes para obtener el reconocimiento y ganancias que esperan las pequeñas casas cerveceras.

Al situarnos en un panorama nacional, existen 940 cervecerías artesanales (ACERMEX, 2019), pero pocas de ellas alcanzarán a tener una identidad debido al gran problema que enfrentan de reconocimiento. Las grandes cervezas industriales nacionales y las cervezas importadas tienen reconocimiento por su lugar de origen y toman ventaja de este. Existen diversas investigaciones, Melewar y Skinner (2018) y Wang et al. (2017), en las que se ha comprobado que marcas extranjeras como Heineken, entre otras de origen europeo, hacen uso de su país de origen para enfatizar su superioridad en la calidad del producto y obtener aceptación social.

Otro de los grandes retos a los que se enfrentan las pequeñas casas productoras, es el precio de venta final de sus productos. Se conoce que el precio de un bien es un factor determinante en la adquisición de un producto. Los costos de producción de cerveza artesanal son elevados 
PANORAMA DE LAS CARACTERÍSTICAS DEL CONSUMIDOR DE CERVEZA ARTESANAL EN LA CIUDAD DE MONTERREY, NUEVO LEÓN.

debido a que, a nivel nacional la mayoría de los insumos que se necesitan para producirla a pequeña escala son importados. Según la ACERMEX, en el 2018 el 47.18\% de las cervecerías dijeron haber usado malta y cereales de origen europeo (2019, p. 20).

Durante el 2017, el INEGI (2017, p. 12) reportó que del gasto total por consumo de bienes y servicios para la elaboración de cerveza el $23.3 \%$ correspondía a materias primas y materiales que se integran a la producción, también que el $20.7 \%$ son gastos en publicidad y servicios de comunicación, y que el 15.1\% representó los gastos por consumo de envases y empaques.

El $71.47 \%$ de los casos, aunque esto represente un gran gasto, las cervecerías artesanales prefieren utilizar envases de vidrio con capacidad de $355 \mathrm{ml}$ por su fácil transportación e incluso para facilitar la venta en lugares tanto en off-trade como on-trade, pero el barril retornable, correspondiente a la preferencia del $25.09 \%$, es el segundo envase seleccionado por los productores (Asociación de Cerveceros Mexicanos, 2019, p. 17). Muchas de las cervecerías artesanales han optado por vender su producto por barril e incluso venderlo dentro de la misma planta productora sin envasar para evitar estos gastos, diversificando así su cartera de negocios.

La venta dentro de las plantas productoras de cerveza artesanal, son una de las múltiples estrategias de marketing que han llevado a cabo estos arriesgados emprendedores. El uso del diseño del envase, la utilización de especias y sabores fuera de lo común, nombres atrevidos, los eventos y experiencias en torno a la cerveza, son algunas de las características que ofrece el mercado de la cerveza artesanal, pero ¿Qué más se puede hacer para aumentar el reconocimiento de una cerveza artesanal?

Solomón (2008, p. 9) menciona, que los datos sobre los consumidores ayudan a las organizaciones a definir el mercado, que el proceso de segmentación del mercado identifica grupos de consumidores que son similares entre sí de una o varias formas, y luego diseña estrategias de marketing que atraigan a uno o más grupos.

\section{MATERIAL Y MÉTODO}

La presente investigación cuenta con un enfoque cuantitativo de diseño no experimental, transversal, de alcance descriptivo y de muestra no probabilística. El tamaño de la muestra en la cual está basado el estudio es de 296, cabe destacar que al ser una muestra no 
PANORAMA DE LAS CARACTERÍSTICAS DEL CONSUMIDOR DE CERVEZA ARTESANAL EN LA CIUDAD DE MONTERREY, NUEVO LEÓN.

probabilística y por conveniencia no es representativa del universo de estudio, son casos seleccionados con características específicas (Hernández, 2014, p. 190).

El instrumento realizado para la recolección de datos fue un cuestionario de 10 preguntas cerradas, el cual fue diseñado para obtener datos demográficos como la edad, género, ocupación, nivel de estudios. También se abordó el factor psicográfico donde se exploraron las conductas del consumidor.

Dicho cuestionario fue aplicado en dispositivos móviles (tableta y teléfono celular) vía electrónica para facilitar la captura de datos y hacer más dinámica la respuesta del encuestado. El estudio se realizó en la ciudad de Monterrey, Nuevo León, dentro de bares/restaurantes especializados en venta de cerveza artesanal, el periodo de aplicación fue del 30 de julio de 2019 al 8 de agosto del mismo año.

Para reducir la ambigüedad del instrumento se realizó una prueba piloto con 60 personas, durante la recolección de respuestas se preguntó acerca de la comprensión de las preguntas y sugerencias para ser evaluadas. Posterior a la evaluación del cuestionario se prosiguió con la recolección de datos.

La encuesta se aplicó con preguntas cerradas y su primera fase se compuso por variables de identificación del consumidor de cerveza artesanal, evaluadas por casillas de verificación; la segunda fase de cuestionario abordó aspectos psicográficos que fueron evaluados en su mayoría en escala de Likert.

\section{RESULTADOS}

De un total de 296, se encuestó a 181 hombres y a 115 mujeres, correspondientes al $61.1 \%$ y $38.9 \%$ respectivamente. Se encontró la existencia de dos grandes grupos de edades que destacaron como consumidores de cerveza artesanal, se logra observar en la Tabla 1, que el mayor porcentaje de los encuestados (46.28\%) se encuentra en un rango de edad de 25 a 35 años; el segundo rango de edad representa al 30.74\% de los encuestados que son menores de 25 años, pero mayores de 18, es de destacar que en el proceso de recolección de los datos la encuesta solo fue aplicada a personas mayores de 18; en menor porcentaje a los rangos pasados, con valor del $11.82 \%$, se encuentran los consumidores dentro de 35 a 45 años; el 
PANORAMA DE LAS CARACTERÍSTICAS DEL CONSUMIDOR DE CERVEZA ARTESANAL EN LA CIUDAD DE MONTERREY, NUEVO LEÓN.

valor porcentual correspondiente a los rangos de edad más pequeños es de $11.15 \%$ que identifica a las personas mayores a 55 años.

Tabla 1

Rango de edad

\begin{tabular}{lcc}
\hline \multicolumn{1}{c}{ Rango de edad } & Frecuencia & Porcentaje \\
\hline $18-25$ & 91 & 30.7 \\
$26-35$ & 137 & 46.3 \\
$36-45$ & 35 & 11.8 \\
$46-55$ & 22 & 7.4 \\
Mayor a 55 & 11 & 3.7 \\
Total & 296 & 100 \\
\hline
\end{tabular}

Fuente: Elaboración propia con datos recabados en julio de 2019 en la ciudad de Monterrey, Nuevo León.

La gran mayoría eran solteros, correspondientes al 76\%. De igual forma, un gran porcentaje se encontraban laborando, con valor de $84.3 \%$ y solo una minoría del 5.2\%, trabajaba y estudiaba a la vez. El nivel de estudios de 182 de los encuestados que son equivalentes al 61.5\%, seguido por el $26.7 \%$, indican que cuentan en un nivel se estudios "superior" y "posgrado", respectivamente.

Dentro del instrumento se evaluó el orden de importancia de factores como el precio, sabor, entre otros, que consideraba el consumidor al momento de adquirir una cerveza artesanal. Como se observa en la Tabla 2, los hallazgos de las respuestas podemos resumirlos de la siguiente manera: el $25.3 \%$ de los consumidores opinan que el precio es el segundo factor al momento de adquirir esta clase de productos, 62.5\% consideran que el sabor es el principal factor determinante de su elección de compra de cerveza artesanal; $29.1 \%$ opinó que la presentación es su segundo factor más relevante; más del 24\% posiciona el grado de alcohol como el tercer factor decisivo para adquirir este producto; el $26.4 \%$ considera los sellos de calidad en quinto, y por último, el factor de importancia para realizar la elección de compra. 
Original

PANORAMA DE LAS CARACTERÍSTICAS DEL CONSUMIDOR DE CERVEZA ARTESANAL EN LA CIUDAD DE MONTERREY, NUEVO LEÓN.

Tabla 2

¿Cuál es el orden de los siguientes factores que consideras al momento de adquirir una cerveza artesanal?

\begin{tabular}{lcccccc}
\hline Factores & \multicolumn{5}{c}{ Resultados en porcentaje \% } & Total \\
\hline & 1 & 2 & 3 & 4 & 5 & \\
\cline { 2 - 6 } Precio & 18.9 & 25.3 & 22 & 15.9 & 17.9 & $100 \%$ \\
Sabor & 62.5 & 10.1 & 4.4 & 4.7 & 18.2 & $100 \%$ \\
Presentación & 14.2 & 29.1 & 25.3 & 18.2 & 13.2 & $100 \%$ \\
Grados de alcohol & 13.2 & 23.4 & 24.4 & 22.7 & 16.3 & $100 \%$ \\
Sellos de calidad & 18.2 & 16.9 & 24.7 & 13.9 & 26.4 & $100 \%$ \\
\hline Fuente: Elaboración propia con datos recabados en julio de 2019 en la ciudad de Monterrey, Nuevo León.
\end{tabular}

Otras características destacables de los consumidores de cerveza artesanal, es la preferencia por presentaciones de 355ml, 205 encuestados correspondientes al 69.3\%, opinaron de esta manera.

\section{Tabla 3}

De acuerdo a su preferencia, ¿Cuál es la mejor presentación de cerveza?

\begin{tabular}{lcc}
\hline Presentación & Frecuencia & Porcentaje \\
\hline $190 \mathrm{ml}$ & 17 & 5.7 \\
$355 \mathrm{ml}$ & 205 & 69.3 \\
$473 \mathrm{ml}$ & 59 & 19.9 \\
$710 \mathrm{ml}$ & 11 & 3.7 \\
Otro & 4 & 1.4 \\
Total & 296 & 100 \\
\hline
\end{tabular}

Fuente: Elaboración propia con datos recabados en julio de 2019 en la ciudad de Monterrey, Nuevo León.

La frecuencia de consumo de 82 encuestados, es al menos una vez al mes, otros 73 de ellos consumen una vez a la semana y 66 lo hacen más de dos veces por semana. Por otra parte, 57 consumidores afirman que adquieren cerveza artesanal solo en ocasiones especiales.

\section{Tabla 4}

¿Qué tan frecuentemente consume cerveza artesanal?

\begin{tabular}{lcc}
\hline Frecuencia de consumo & Frecuencia & Porcentaje \\
\hline Más de 2 veces por semana & 66 & 22.3 \\
1 vez a la semana & 73 & 24.7 \\
1 vez al mes & 82 & 27.7 \\
1 vez cada dos meses & 18 & 6.1 \\
Sólo en ocasiones especiales & 57 & 19.3 \\
Total & 296 & 100 \\
\hline
\end{tabular}

Fuente: Elaboración propia con datos recabados en julio de 2019 en la ciudad de Monterrey, Nuevo León. 
PANORAMA DE LAS CARACTERÍSTICAS DEL CONSUMIDOR DE CERVEZA ARTESANAL EN LA CIUDAD DE MONTERREY, NUEVO LEÓN.

En la siguiente tabla, se puede observar cuántas piezas consumen por cada ocasión, el 43.2\% de los encuestados dijo adquirir de una a dos piezas; otro $44.3 \%$ beben entre tres a cuatro piezas. Solo una minoría, consume más de cinco piezas por cada ocasión.

\section{Tabla 5}

¿Cuántas cervezas artesanales consume regularmente?

\begin{tabular}{lcr}
\hline No. de piezas & Frecuencia & Porcentaje \\
\hline De 1 a 2 & 128 & 43.2 \\
De 3 a 4 & 131 & 44.3 \\
De 5 o más & 37 & 12.5 \\
Total & 296 & 100 \\
\hline
\end{tabular}

Fuente: Elaboración propia con datos recabados en julio de 2019 en la ciudad de Monterrey, Nuevo León.

Un gran porcentaje de encuestados, son consumidores de cerveza artesanal hace más de tres años (39.9\%), los consumidores relativamente nuevos solo corresponden a un $12.8 \%$.

\section{Tabla 6}

¿Cuánto tiempo tienes consumiendo cerveza artesanal?

\begin{tabular}{lcc}
\hline Tiempo & Frecuencia & Porcentaje \\
\hline Menos de un año & 38 & 12.8 \\
Un año & 31 & 10.5 \\
Dos años & 60 & 20.3 \\
Tres años & 49 & 16.6 \\
Más de tres años & 118 & 39.9 \\
Total & 296 & 100 \\
\hline
\end{tabular}

Fuente: Elaboración propia con datos recabados en julio de 2019 en la ciudad de Monterrey, Nuevo León.

El $45.9 \%$ de los participantes, conocieron la cerveza artesanal por un amigo, seguido por el $27 \%$ que representan a las personas que conocieron este producto en un festival de cerveza. Los encuestados que marcaron "otro" (18.9\%) como medio por el cual conocieron la cerveza artesanal coincidieron en haberla conocido debido a su trabajo.

\section{Tabla 7}

De las siguientes opciones, seleccione en cuál conoció la cerveza artesanal

\begin{tabular}{lcc}
\hline Opciones & Frecuencia & Porcentaje \\
\hline En un festival de cerveza & 80 & 27 \\
Por un amigo & 136 & 45.9 \\
Publicidad en YouTube, & 11 & 3.7 \\
Facebook e Instagram & 13 & 4.4 \\
Medios impresos & 56 & 18.9 \\
Otra & 296 & 100 \\
Total &
\end{tabular}

Fuente: Elaboración propia con datos recabados en julio de 2019 en la ciudad de Monterrey, Nuevo León. 
PANORAMA DE LAS CARACTERÍSTICAS DEL CONSUMIDOR DE CERVEZA ARTESANAL EN LA CIUDAD DE MONTERREY, NUEVO LEÓN.

El instrumento aplicado tomó en cuenta la importancia que representaba el consumo de cerveza artesanal como un estilo de vida, los resultados de la encuesta fueron a favor, un porcentaje de 32.4 no especifica que "consumir cerveza artesanal como un estilo de vida no representa nada" pero tampoco afirma lo contrario. Por otro lado, encontramos que el $26.4 \%$ afirma que consumir este producto no representa un estilo de vida para ellos. De la misma manera, se analizó que por arriba del 27\% consideran que consumir este producto es un momento para socializar, sin dejar de lado que el $39.9 \%$ piensan que consumir cerveza artesanal es un momento para relajarse y consentirse.

Tabla 8

¿Qué representa para usted consumir cerveza artesanal?

\begin{tabular}{|c|c|c|c|c|c|c|c|c|c|c|c|}
\hline \multirow[t]{3}{*}{ Indicadores } & \multicolumn{10}{|c|}{ Resultados } & \multirow[t]{2}{*}{ Total } \\
\hline & \multicolumn{2}{|c|}{ Nada } & \multicolumn{2}{|c|}{ Casi nada } & \multicolumn{2}{|c|}{ Poco } & \multicolumn{2}{|c|}{ Mucho } & \multicolumn{2}{|c|}{ Demasiado } & \\
\hline & Frecuencia & Porcentaje & Frecuencia & Porcentaje & Frecuencia & Porcentaje & Frecuencia & Porcentaje & Frecuencia & Porcentaje & \\
\hline $\begin{array}{l}\text { Un estilo de } \\
\text { vida }\end{array}$ & 78 & 26.4 & 39 & 13.2 & 96 & 32.4 & 47 & 15.9 & 36 & 12.2 & $100 \%$ \\
\hline $\begin{array}{l}\text { Un momento } \\
\text { para socializar }\end{array}$ & 39 & 13.2 & 41 & 13.9 & 70 & 23.6 & 82 & 27.7 & 64 & 21.6 & $100 \%$ \\
\hline $\begin{array}{l}\text { Un momento } \\
\text { para relajarse } \\
\text { y consentirse }\end{array}$ & 40 & 13.5 & 26 & 8.8 & 53 & 17.9 & 59 & 19.9 & 118 & 39.9 & $100 \%$ \\
\hline
\end{tabular}

Fuente: Elaboración propia con datos recabados en julio de 2019 en la ciudad de Monterrey, Nuevo León.

Dado que existen diversos espacios que comparten conocimientos sobre el producto en cuestión, el cuestionario evaluó la manera en la que se informan los consumidores. Los hallazgos de las respuestas en torno a la manera en que se informan son los siguientes: el $17.3 \%$ ocasionalmente se informa en blogs de cerveza artesanal; el 14.2\% siempre se informa en los blogs de cerveza y el $41.7 \%$ nunca lo hace en este medio.

Maltap, es la plataforma mexicana que informa acerca de los estilos cerveceros y nuevas etiquetas, cuenta con ubicación en tiempo real para recomendarte los puntos de venta de este bien, entre otras múltiples funciones. Podría resumirse que arriba del $30 \%$ conocen está aplicación, de los cuales el 9.8\% siempre la consultan y el 59.1\% la desconoce por completo. Al igual que la aplicación anterior, existe Untappd que, aunque es extranjera es relativamente más utilizada. 
PANORAMA DE LAS CARACTERÍSTICAS DEL CONSUMIDOR DE CERVEZA ARTESANAL EN LA CIUDAD DE MONTERREY, NUEVO LEÓN.

Los canales de YouTube especializados en informar sobre cerveza artesanal son otra fuente conocida por el consumidor, puede decirse que son más consultados después de los blogs y que por arriba del 50\% de los encuestados se han informado por este medio, siendo el $12.5 \%$ consumidores que siempre lo toman como un referente.

Las revistas de cerveza artesanal, son las más consultadas de manera ocasional, el 16.9\% contestó de esa manera, el 10.5\% respondió que se informan siempre por este medio y el $50.7 \%$ nunca lo hacen.

Organismos como Cerveceros Mexicanos y ACERMEX, en apoyo para el crecimiento de la industria, difunden en medios digitales la cultura cervecera por medio de páginas web y aplicaciones tanto privadas como gubernamentales, y son un referente para más del $50 \%$ de los participantes, de los cuales el $14.9 \%$ las consultan de manera ocasional y un $13.9 \%$ lo hace siempre.

\section{Tabla 9}

¿Cuál es la manera en la que se informa acerca de la cerveza artesanal?

\begin{tabular}{|c|c|c|c|c|c|c|c|c|c|c|c|}
\hline \multirow[t]{2}{*}{ Indicadores } & \multicolumn{2}{|c|}{ Nunca } & \multicolumn{2}{|c|}{ Raramente } & \multicolumn{2}{|c|}{ Ocasionalmente } & \multicolumn{2}{|c|}{ Casi siempre } & \multicolumn{2}{|c|}{ Siempre } & \multirow[t]{2}{*}{ Total \% } \\
\hline & Frecuencia & Porcentaje & Frecuencia & Porcentaje & Frecuencia & Porcentaje & Frecuencia & Porcentaje & Frecuencia & Porcentaje & \\
\hline $\begin{array}{l}\text { Blogs de cerveza } \\
\text { artesanal }\end{array}$ & 123 & 41.7 & 40 & 13.6 & 51 & 17.3 & 39 & 13.2 & 42 & 14.2 & $100 \%$ \\
\hline Maltap & 175 & 59.1 & 37 & 12.5 & 36 & 12.2 & 19 & 6.4 & 29 & 9.8 & $100 \%$ \\
\hline Untappd & 170 & 57.6 & 38 & 12.9 & 40 & 13.6 & 19 & 6.4 & 28 & 9.5 & $100 \%$ \\
\hline $\begin{array}{l}\text { Canales de } \\
\text { YouTube sobre } \\
\text { cerveza artesanal }\end{array}$ & 141 & 47.6 & 36 & 12.2 & 44 & 14.9 & 38 & 12.8 & 37 & 12.5 & $100 \%$ \\
\hline $\begin{array}{l}\text { Revistas de } \\
\text { cerveza artesanal }\end{array}$ & 150 & 50.7 & 41 & 13.9 & 49 & 16.9 & 25 & 8.4 & 31 & 10.5 & $100 \%$ \\
\hline $\begin{array}{l}\text { Paginas oficiales } \\
\text { en linea } \\
\text { (ACERMEX, etc). }\end{array}$ & 142 & 48 & 38 & 12.8 & 44 & 14.9 & 31 & 10.5 & 41 & 13.9 & $100 \%$ \\
\hline
\end{tabular}

Fuente: Elaboración propia con datos recabados en julio de 2019 en la ciudad de Monterrey, Nuevo León.

Los consumidores difieren en cuanto a preferencias del lugar de consumo, por tanto, se identificaron algunos puntos de venta y se evaluaron individualmente. La venta de cerveza artesanal en supermercados a nivel local es cada vez más regular y $20.9 \%$ de los encuestados marcaron que siempre adquieren sus cervezas en estos espacios. 
PANORAMA DE LAS CARACTERÍSTICAS DEL CONSUMIDOR DE CERVEZA ARTESANAL EN LA CIUDAD DE MONTERREY, NUEVO LEÓN.

Debido a que las grandes casas cerveceras están adquiriendo cervecerías artesanales o realizando alianzas con las mismas, ver la oferta de productos más variada en los depósitos ha llevado a la evaluación de compra del consumidor en este tipo de puntos de venta. El $45.6 \%$ ha respondido que nunca ha adquirido esta clase de producto en depósitos y otro $18.2 \%$ lo hace de manera ocasional.

El e-commerce facilita la venta de productos de cualquier tipo, por lo cual no es raro que algunas cervecerías lo utilicen para comercializar esta bebida alcohólica. Puede observarse, que un $16.5 \%$ siempre prefiere adquirir los productos en estas plataformas, el $20.7 \%$ lo hace ocasionalmente y un $35.5 \%$ nunca.

Los restaurantes son otro tipo de puntos de venta donde se puede adquirir una cerveza de esta categoría y es el segundo lugar de preferencia para adquirirlas "siempre", con un valor de $44.9 \% \mathrm{y}$, en primer lugar, se encuentran los bares, que corresponden al $61.1 \%$ de los consumidores.

\section{Tabla 10}

¿En cuál de las siguientes opciones prefiere adquirir cervezas artesanales?

\begin{tabular}{|c|c|c|c|c|c|c|c|c|c|c|c|}
\hline \multirow[t]{2}{*}{ Indicadores } & \multicolumn{2}{|l|}{ Nunca } & \multicolumn{2}{|c|}{ Raramente } & \multicolumn{2}{|c|}{ Ocasionalmente } & \multicolumn{2}{|c|}{ Casi siempre } & \multicolumn{2}{|c|}{ Siempre } & \multirow[t]{2}{*}{ Total \% } \\
\hline & Frecuencia & Porcentaje & Frecuencia & Porcentaje & Frecuencia & Porcentaje & Frecuencia & Porcentaje & Frecuencia & Porcentaje & \\
\hline Supermercados & 80 & 27 & 49 & 16.6 & 54 & 18.2 & 51 & 17.2 & 62 & 20.9 & $100 \%$ \\
\hline Depósitos & 135 & 45.6 & 51 & 17.2 & 54 & 18.2 & 25 & 8.4 & 31 & 10.5 & $100 \%$ \\
\hline $\begin{array}{l}\text { Plataformas } \\
\text { oficiales }\end{array}$ & 104 & 35.3 & 38 & 12.9 & 61 & 20.7 & 43 & 14.6 & 49 & 16.6 & $100 \%$ \\
\hline Restaurantes & 33 & 11.1 & 27 & 9.1 & 35 & 11.8 & 68 & 23 & 133 & 44.9 & $100 \%$ \\
\hline Bar & 24 & 8.1 & 11 & 3.7 & 29 & 9.8 & 51 & 17.2 & 181 & 61.1 & $100 \%$ \\
\hline
\end{tabular}

Fuente: Elaboración propia con datos recabados en julio de 2019 en la ciudad de Monterrey, Nuevo León.

\section{DISCUSIÓN Y CONCLUSIÓN}

Puede asumirse que el primer paso para que una cervecería artesanal pueda crecer en la industria mexicana es ofertar un buen producto, debido a que el segmento se encuentra dispuesto a adquirirlo principalmente por el sabor. En virtud de lo anterior, el marketing resulta ser una buena herramienta para generar un exitoso primer contacto con el consumidor.

Cabe destacar que, aunque existan diversas investigaciones sobre el producto "cerveza artesanal", el segmento y las características que posea pueden variar dependiendo de los 
PANORAMA DE LAS CARACTERÍSTICAS DEL CONSUMIDOR DE CERVEZA ARTESANAL EN LA CIUDAD DE MONTERREY, NUEVO LEÓN.

aspectos de su entorno tales como los factores culturales y económicos, entre otros. En el caso de la presente investigación, los datos proporcionados, podrán ayudar a crear estrategias de marketing focalizadas en el consumidor de la ciudad de Monterrey, permitiendo a la cervecería tipo artesanal obtener reconocimiento principalmente a nivel local.

Dentro de los datos analizados, se encontraron características demográficas y psicográficas, que ayudan a perfilar e identificar al consumidor de cerveza artesanal. Por lo tanto, en la ciudad de Monterrey, el segmento que mayormente adquiere este producto se encuentra en un rango de edad de 18 a 35 años en su etapa de soltería, en su mayoría hombres, mismos que laboran actualmente y se encuentran en un nivel superior de estudios.

Al asumir la relación que existe entre el nivel de estudios en el que se encuentra el $88.2 \%$ de los encuestados y la ocupación del $84 \%$ ellos, esta relación puede ser un indicador de factores como un mayor nivel de ingresos, debido a un alto nivel de estudios e incluso justificar el por qué el factor precio no es lo más importante a la hora de consumir una cerveza artesanal.

Pese a que $76.8 \%$ de los encuestados son conocedores de esta bebida artesanal desde hace más de 2 años, solo un pequeño porcentaje considera el consumo como un estilo de vida que se informa constantemente o que se involucra a un nivel superior con este tipo de bebidas. Es importante diferenciar que, aunque un porcentaje pequeño se identifique de está manera, el producto cerveza artesanal en sí se encuentra en un ciclo de vida de estilo, como hacen mención Kotler y Keller (2012, p. 310), esto se refiere a que en ocasiones su consumo aumente en mayor medida al estar a la moda en ciertos periodos.

La popularidad del consumo de la cerveza artesanal ha sido en primera instancia por la recomendación de un buen amigo o la publicidad de boca en boca, este dato es de vital importancia pues en otras investigaciones como la de Carvajal y Zapata (2012, p. 114), también se hace referencia a la influencia de la opinión de los amigos, que es un factor que incide en la decisión final del consumo de cerveza.

Los mayores esfuerzos de marketing han sido la visualización y venta de esta clase de productos en festivales y ferias, lo cual se ve reflejado en las respuestas del $27 \%$ de los encuestados, que afirman haber tenido un primer contacto con este producto en dicho lugar. Robin, Martínez Astorga y Valencia (2017, p. 22) a través de su estudio recomiendan que "las fiestas y ferias de cerveza artesanal son una instancia de la cual los productores no debiesen MARTÍNEZ-SILVA M. DEL S., SÁNCHEZ-ROSADO O. B. 
PANORAMA DE LAS CARACTERÍSTICAS DEL CONSUMIDOR DE CERVEZA ARTESANAL EN LA CIUDAD DE MONTERREY, NUEVO LEÓN.

ausentarse". Otro lugar para considerar son bares y restaurantes, los resultados indican que son los lugares preferentes para consumir esta bebida milenaria.

No obstante, al contar con limitaciones de distribución debido a un bajo presupuesto e incluso aspectos legales, hay que tener en consideración otros canales actuales, como lo sería el contar con una plataforma online, lo cual facilita al consumidor conocer y adquirir el producto cerveza artesanal. Este canal no es desconocido para este consumidor, pues un porcentaje considerable señaló que ocasionalmente adquiere el producto por este medio. Acompañar el producto con servicios pick up o envíos a domicilio, pueden ser un factor clave al destacar sobre otras cervecerías de este tipo.

Debido a las pocas posibilidades de jugar con el factor precio como un atractivo para el consumidor, optar por otras estrategias de comercialización sería lo recomendado. Competir entre cervecerías artesanales y que las mismas marquen las diferencias contra las industriales, el crecimiento del mercado, y la exclusividad de este, ayudará a que sean sinónimo de productos únicos en el amplio mercado de cervezas y bebidas alcohólicas.

En la capital del estado de Nuevo León, la preferencia por el consumo de cerveza industrial no ha sido una limitante para demostrar que existe un segmento dispuesto a degustar un producto con identidad local. El mercado de cerveza artesanal ha ido creciendo en Monterrey y en otras entidades de la república mexicana, hoy en día, las grandes cervecerías están dispuestas a adquirir y realizar alianzas con pequeñas marcas cerveceras, para continuar satisfaciendo las necesidades de este exigente consumidor.

\section{REFERENCIAS BIBLIOGRÁFICAS}

Asociación de Cerveceros Mexicanos. (2019). Reporte de la industria cervecera independiete mexicana 2018. http://acermex.org/wp-content/uploads/2020/01/Reporte-de-la-Industria-.Acermex-2actua.pdf

Calvillo, E. (2017). La Cerveza Artesanal Una experiencia multisensorial. En Deloitte. https://www2.deloitte.com/content/dam/Deloitte/mx/Documents/consumerbusiness/2017/Cerveza-Artesanal-Mexico-2017.pdf 
PANORAMA DE LAS CARACTERÍSTICAS DEL CONSUMIDOR DE CERVEZA ARTESANAL EN LA CIUDAD DE MONTERREY, NUEVO LEÓN.

Carvajal, J. \& Zapata, E. (2012). Análisis De Percepciones De Consumidores De Bebidas Alcohólicas - Productos Cerveceros. Criterio Libre, 10(17), 107-126. https://doi.org/10.18041/1900-0642/criteriolibre.17.1143

Contreras, C. \& Ortega, I. (2005). Bebidas y regiones: historia e impacto de la cultura etílica en México (Plaza y valdes (Ed.). https://books.google.es/books?hl=es\&lr=\&id=N69XIPt_Pe8C\&oi=fnd\&pg=PA85\&dq=cervec eria+cuauhtémoc\&ots=B8xSSyFy9Y\&sig=tqpnwaQzT⿳亠丷厂ec-

4XdicsWR4jh6GM\#v=onepage \&q=cerveceria cuauhtémoc\&f=false

Hernández, R. (2014). Metodología de la investigación. (6ª. ed.). México: McGraw-Hill.

Instituto Nacional de Estadística y Geografía. (2017). La actividad de elaboración de cerveza. https://www.inegi.org.mx/app/biblioteca/ficha.html?upc=702825096649

Kantar World Panel. (2016). México $2^{\circ}$ lugar en consumo de Cerveza en LatAm. https://www.kantarworldpanel.com/mx/Noticias-/Mexico-2-lugar-en-consumo-de-Cervezaen-Latinoamerica

Kotler, P. \& Keller, K. (2012). Direccion de marketing. En Pearson Educación (14a ed., Vol. 1). Pearson Educación. https://doi.org/10.1017/CBO9781107415324.004

Melewar, T. \& Skinner, H. (2018). Beer consumption and perceptions of Utopic authenticity. En The Institute of Place Management (Ed.), 5th Corfu Symposium on Managing \& Marketing Places (p. 4). The Institute of Place Management.

Murray, D. W. \& O'Neill, M. A. (2012). Craft beer: Penetrating a niche market. British Food Journal, 114(7). https://doi.org/10.1108/00070701211241518

Ortega Ridaura, I. (2002). Cervecería Cuauhtémoc expansión y financiamiento (1890-1982 ). Entorno económico, XL(236), 8. http://eprints.uanl.mx/8697/1/Cerveceria Cuauhtemoc.pdf

¿Qué es una cerveza artesanal? (2018). Asociación de Cerveceros Mexicanos. https://acermex.org/cerveza-artesanal/

Quiroga, M. (2018). Cerveza producida en México: orgullo nacional. Forbes. https://www.forbes.com.mx/cerveza-producida-en-mexico-orgullo-nacional/

Recio, G. (2004). El nacimiento de la industria cervecera en México, 1880-1910. Segundo Congreso Nacional de Historia Económica, 46.

Robin, C. F., Martínez, D. Y., Astorga, P. S. \& Valencia, J. C. (2017). Comportamiento del Consumidor de Cerveza Artesanal. Revista global de negocios, 5(1), 19-22. http://www.theibfr2.com/RePEc/ibf/rgnego/rgn-v5n1-2017/RGN-V5N1-2017.pdf\#page=19

Secretaría de Salud \& Instituo Nacional de Salud Pública Instituto Nacional de Estadística y Geografía. (2018). Encuesta Nacional de Salud y Nutrición. En Ensanut (Vol. 1). https://doi.org/10.1017/CBO9781107415324.004

Solomon, M. R. (2008). Comportamiento del consumidor (P. M. Guerrero (Ed.); (7ª ed.). México: 
PANORAMA DE LAS CARACTERÍSTICAS DEL CONSUMIDOR DE CERVEZA ARTESANAL EN LA CIUDAD DE MONTERREY, NUEVO LEÓN.

\section{Pearson Educación.}

Suárez, M. (2013). Cerveza: componentes y propiedades. En Procesos de fabricación de bebidas alcohólicas. http://digibuo.uniovi.es/dspace/bitstream/10651/19093/8/TFM_ Maria Suarez Diaz.pdf

Urrutia, B. A., Aguilar, J. \& Muñoz, M. (2016). Formación de redes estrategicas para crear valor: las cervecerías artesanales en México. Revista Mexicana de Agronegocios, 39, 377-388. https://www.redalyc.org/pdf/141/14149188002.pdf

Villezca, P. (2005). Análisis del consumo de cerveza en el área metropolitana de Monterrey. Un modelo de respuesta censurada. Ciencia UANL, VIII(3), 339-345. https://www.redalyc.org/pdf/402/40280306.pdf

Wang, O., Gellynck, X., \& Verbeke, W. (2017). Chinese consumers and European beer: Associations between attribute importance, socio-demographics, and consumption. Appetite, 108, 416-424. https://doi.org/https://doi.org/10.1016/j.appet.2016.10.029

Workman, D. (2020). Beer Exports by Country. Worldstopexports. http://www.worldstopexports.com/beer-exports-by-country/ 